\title{
Biochemical characterization of a recombinant SARS coronavirus nsp12 RNA-dependent RNA polymerase capable of copying viral RNA templates
}

\author{
Dae-Gyun Ahn · Jin-Kyu Choi $\cdot$ Deborah R. Taylor • \\ Jong-Won Oh
}

Received: 7 February 2012/ Accepted: 24 May 2012/Published online: 13 July 2012

(C) Springer-Verlag 2012

\begin{abstract}
The severe acute respiratory syndrome coronavirus (SARS-CoV) RNA genome is replicated by a virus-encoded RNA replicase, the key component of which is the nonstructural protein 12 (nsp12). In this report, we describe the biochemical properties of a full-length recombinant SARS-CoV nsp12 RNA-dependent RNA polymerase (RdRp) capable of copying viral RNA templates. The purified SARS-CoV nsp12 showed both primerdependent and primer-independent RNA synthesis activities using homopolymeric RNA templates. The RdRp activity was strictly dependent on $\mathrm{Mn}^{2+}$. The nsp12 preferentially copied homopolymeric pyrimidine RNA templates in the absence of an added oligonucleotide primer. It was also able to initiate de novo RNA synthesis from the 3 '-ends of both the plus- and minus-strand genome of SARS-CoV, using the 3'-terminal 36- and 37-nt RNA, respectively. The in vitro RdRp assay system established with a full-length nsp12 will be useful for understanding the mechanisms of coronavirus replication and for the development of anti-SARS-CoV agents.
\end{abstract}

D.-G. Ahn · J.-K. Choi · J.-W. Oh ( $₫)$

Department of Biotechnology, Yonsei University, 134 Shinchon-dong, Seodaemun-gu, Seoul 120-749, Korea e-mail: jwoh@yonsei.ac.kr

\section{R. Taylor}

Laboratory of Emerging Pathogens, Division of Emerging and Transfusion Transmitted Diseases, Office of Blood Research and Review, Center for Biologics Evaluation and Research, Food and Drug Administration, Bethesda, MD 20892, USA

J.-W. Oh

Translational Research Center for Protein Function Control, Yonsei University, 134 Shinchon-dong, Seodaemun-gu, Seoul 120-749, Korea

\begin{abstract}
Abbreviations
SARS-CoV Severe acute respiratory syndrome coronavirus

RdRp RNA-dependent RNA polymerase

Nsp12 Nonstructural protein 12
\end{abstract}

\section{Introduction}

Severe acute respiratory syndrome coronavirus (SARS-CoV) belongs to the genus Betacoronavirus of the family Coronaviridae and has a plus-strand RNA genome of approximately $29.7 \mathrm{~kb}$ with a 5' cap-structure and a 3' poly(A) tail [18]. The SARS-CoV genome has 14 open reading frames (ORFs) flanked by 5' and 3' untranslated regions (UTRs), which are essential cis-acting elements required for viral replication [29]. The 5' two-thirds of the viral genome contains two large ORFs, $1 \mathrm{a}$ and $1 \mathrm{~b}$, the latter of which is expressed by ribosomal frameshifting near the 3' end of ORF1a [1,23]. The two large polyproteins, ppla, encoded by ORF1a, and pp1ab encoded by ORF1a and ORF1b, are processed by two viral proteases to generate a total of 16 nonstructural proteins (nsps), most of which likely serve as RNA replicase components $[29,35]$. The nsp12, which is the first protein encoded by ORF1b, has common viral RNA-dependent RNA polymerase (RdRp) motifs and is thus the catalytic core of the RNA replicase [39].

RNA genome replication is a crucial step in SARS-CoV propagation and is mediated by the RNA replicase, which has been proposed to be comprised of viral nsps and host proteins. The SARS-CoV replicase complex transcribes the full-length plus-strand viral genome and its complementary minusstrand genomic RNA template. In addition, the replication complex transcribes a set of 3'-coterminal nested subgenomic mRNAs with a 5' leader sequence derived from the 5', 
end of the genome and the corresponding minus-strand subgenomic RNAs by discontinuous RNA transcription [26, 29]. During synthesis of the viral genome and its subgenomes, nsp12, alone or with the help of viral and/or cellular proteins, is thought to initiate RNA synthesis from the 3' ends of the viral genome and subgenomes in both the plus and minus senses [37]. Correct initiation of RNA synthesis is essential for maintenance of the integrity of viral genomes. RNA viruses use two principally different mechanisms by which RNA synthesis can be initiated: de novo initiation and primerdependent initiation [12]. During de novo initiation, the starting nucleotide provides the 3' hydroxyl group for the addition of the next nucleotide. In contrast, primer-dependent initiation requires the use of a separate oligonucleotide or protein primer to provide the hydroxyl nucleophile. The mechanisms of RNA synthesis initiation for SARS-CoV have not been well characterized. In particular, the details of subgenomic RNA synthesis remain unknown [19].

An in vitro $\mathrm{RdRp}$ assay system for SARS-CoV would be useful for studying the mechanisms of viral RNA replication, screening for RdRp inhibitors, and identifying target sites of nucleic-acid-based antiviral agents through mapping of important cis-acting elements. Further, it would be an important tool for determining the mode of RNA synthesis initiation to provide important mechanistic insight into the SARS-CoV replication cycle [19]. Previously, the RdRp activity of SARS-CoV nsp12 was demonstrated using nonviral RNA templates $[4,33]$. An RdRp assay system using an enzymatically active nsp12 accepting exogenous viral genome-derived RNA templates would enable us to characterize RNA synthesis initiation mechanisms and to initiate exploration of the mechanisms involved in subgenomic RNA synthesis. However, previous studies did not investigate whether nsp12 uses the 3'-end RNA regions of the viral genome and its complementary minus-strand RNA template.

In the present study, we cloned and expressed a full-length recombinant SARS-CoV nsp12. With the purified recombinant nsp12, we established an in vitro RdRp assay system and demonstrated that nsp12 was capable of de novo RNA synthesis initiation using viral RNA templates corresponding to the 3' ends of the plus and minus strands of the SARS-CoV genome. Furthermore, we mapped the minimal cis-acting regions required for initiation of plus- and minus-strand viral RNA synthesis.

\section{Material and methods}

Construction of the recombinant SARS-CoV nsp12 expression vector

Total RNA from Vero cells infected with the SARS-CoV Urbani strain (GenBank accession number: AY278741) was isolated using TRIzol Reagent (Invitrogen). The RNA was reverse-transcribed using ImProm-II reverse transcriptase (Promega) and the reverse primer Spol_R (5'-C GGGATCCTACTGCAAGACTGTATGTGG-3'; BamHI site underlined). The full-length nsp12 coding sequence was amplified by PCR with Pfu polymerase (Takara), using the forward primer Spol_F (5'-GCTCTAGAATGCAGTC TGCGGATGCATCAACGTTCTTGAATCGGGTTTGCG GTGTAAGTGCAGCCCGT-3'; $\mathrm{XbaI}$ site underlined) and the Spol_R primer. The PCR product was digested with $X b a \mathrm{I}$ and BamHI and ligated into NheI- and BamHIdigested pTrcHisB (Invitrogen) vector to construct the nsp12-expression plasmid pTrcSARSnsp12_FL. The coding sequence of the unique domain $\left(\mathrm{S}_{4370^{-}} \mathrm{E}_{4739}\right)$ of nsp12 was amplified by PCR with the Spol_F and the SpolUnique_R (5'-CGGGATCCCTATTCCTTGAAACTGAG ACGCGAG-3'; BamHI site underlined) primers. The PCR product was ligated into pTrcHisB, generating the construct designated pSARSnsp12_UD. The SDD motif in the nsp12 active site was mutated to SAA by bridged PCR using oligonucleotides containing the target mutations as described previously [21].

Expression and purification of recombinant SARS-CoV nsp12

SARS-CoV nsp12 was expressed in E. coli TOP10 cells (Invitrogen). Cells transformed with pTrcSARSnsp12_FL were grown at $37{ }^{\circ} \mathrm{C}$ to an optical density at $600 \mathrm{~nm}$ of 0.6-0.8. Expression of nsp12 was induced with $0.5 \mathrm{mM}$ isopropyl- $\beta$-D-thiogalactopyranoside for $20 \mathrm{~h}$ at $18^{\circ} \mathrm{C}$. SARS-CoV nsp12 was purified by metal affinity chromatography using Ni-nitrilotriacetic acid-agarose (QIAGEN) resin as described previously [13]. SARS-CoV nsp12containing fractions were collected, dialyzed against buffer A $(50 \mathrm{mM}$ Tris- $\mathrm{HCl}, \mathrm{pH} 8.0,50 \mathrm{mM} \mathrm{NaCl}, 1 \mathrm{mM}$ DTT, $10 \%$ glycerol), and then applied to a Q-Sepharose column (Amersham Biosciences). Bound proteins were eluted with a step-gradient of $\mathrm{NaCl}$ from $50 \mathrm{mM}$ to $800 \mathrm{mM}$. Fractions containing nsp12 were further purified using a Hiload 16/60 Superdex 200 column (Amersham Pharmacia Biotech) as described previously [13]. The SARS-CoV nsp12containing fractions were mixed with cold glycerol $(10 \%$ final concentration) and stored at $-80{ }^{\circ} \mathrm{C}$.

Western blot analysis

Purified SARS-CoV nsp12 and its derivatives were separated by $10 \%$ SDS-PAGE and transferred to a nitrocellulose membrane (Hybond-ECL; GE Healthcare Life Sciences) for probing with an anti-penta (His)-tag antibody (Qiagen) as described previously [13]. 
RNA template preparation

The DNA templates for in vitro RNA transcription were obtained by RT-PCR amplification using the total RNA extracted from SARS-CoV infected cells. The DNA template for the 3'-UTR of the SARS-CoV genome was amplified with the reverse primer 3'-UTR_R (5'-GTC ATTCTCCTAAGAAGCT-3') and the forward primer 3'UTR339_F (5'-TAATACGACTCACTATAGGCCCCA TGTGATTTTAATAGC-3'; the T7 promoter sequence is underlined and the extra $\mathrm{G}$ nucleotide in italics was added for efficient transcription by T7 RNA polymerase) using the cDNA synthesized with the reverse primer 3'-UTR_R. The DNA template for the region complementary to the SARS-CoV 5'-UTR genome (c5'-UTR) was amplified with the reverse primer 5'-UTR_R (5'-TAATACGACT CACTATAGGCTTACCTTTCGGTCACAC-3'; the T7 promoter and extra $\mathrm{G}$ nucleotides are indicated, as described above) and the forward primer 5'UTR_F (5'-ATA TTAGGTTTTTACCTAC-3') using the cDNA synthesized with the reverse primer 5'-UTR_R. DNA templates for deletion derivatives of the 3'-UTR and c5'-UTR were similarly amplified with appropriate sets of primers. The resulting PCR products containing the $\mathrm{T} 7$ promoter were used directly for in vitro transcription using a T7 MEGAscript kit (Ambion) as described previously [1]. DNA templates for the 3'-UTR containing the poly(A) tail was amplified by RT-PCR with the reverse primer 3'UTRBsaI_R (5'-GGTCTCT $\downarrow$ TTTTTTTTTTTTTTTTT-3'; a BsaI site is underlined, and the digestion site is indicated by the arrow) and the forward primer 3'-UTR339_F. PCR products were cloned into pCR2.1TOPO vector (Invitrogen). The resulting plasmids with inserts containing different lengths of poly(A) tail $(14,17,19$, and $33 \mathrm{nt})$ were isolated by sequence analysis. These plasmids, linearized by BsaI digestion, were then used for in vitro transcription to prepare the 3'-UTR templates containing various lengths of poly(A) tail (13, 16, 18, and $32 \mathrm{nt})$.

Prediction of RNA secondary structure

RNA secondary structures were predicted with the RNA mfold program [11]. The structure drawings were edited and annotated using the RnaViz 2 program [6].

\section{RdRp assay}

The in vitro RdRp assay was performed with 3 pmol of recombinant SARS-CoV nsp12 in a total volume of $25 \mu \mathrm{l}$ $\mathrm{RdRp}$ reaction buffer $(50 \mathrm{mM}$ Tris- $\mathrm{HCl}, \mathrm{pH} 8.0,50 \mathrm{mM}$ $\mathrm{NaCl}, 2 \mathrm{mM} \mathrm{MnCl}_{2}$ unless otherwise specified, $1 \mathrm{mM}$ DTT, $10 \%$ glycerol, 20 units of RNase inhibitor [Promega]) containing $1 \mu \mathrm{g}$ of each of the homopolymeric
RNA templates, $5 \mu \mathrm{M}$ complementary rNTP, $10 \mathrm{pmol}$ 20 -mer ribo-oligonucleotide $(\mathrm{rN})_{20}$ primer, and $5 \mu \mathrm{Ci}$ $\left[\alpha-{ }^{32} \mathrm{P}\right]$ of each complementary rNTP $(3,000 \mathrm{Ci} / \mathrm{mmol}$, Amersham Pharmacia Biotech). The reaction mixture was incubated for $2 \mathrm{~h}$ at $32{ }^{\circ} \mathrm{C}$. For RdRp reactions with viral RNA templates, $500 \mathrm{ng}$ RNA template was added to the reaction mixture as described above, except that a mixture of cold ribonucleotides $(500 \mu \mathrm{M}$ each ATP, CTP, and GTP, and $5 \mu \mathrm{M}$ UTP) was included in the reaction with $5 \mu \mathrm{Ci}\left[\alpha-{ }^{32} \mathrm{P}\right]$ UTP. After the RdRp reaction, reaction products were processed and resolved on denaturing $5 \%$ polyacrylamide gels containing $8 \mathrm{M}$ urea as described previously [13]. After electrophoresis, the gels were stained with ethidium bromide to locate the template positions, photographed, dried, and then exposed to X-ray film for autoradiography.

Protein identification by tandem mass spectrometry (MS/MS)

Gel bands were excised and subjected to in-gel trypsin digestion as described previously [28]. Digested samples were desalted using a homemade $\mathrm{C}_{18}$ nano-column. MS/ MS was performed using nano-ESI on a Q-TOF2 mass spectrometer (AB Sciex Instruments). To identify the peptides, all MS/MS spectra recorded for tryptic peptides were searched against protein sequences from NCBInr and EST databases using the MASCOT search program (http:// www.matrixscience.com).

\section{Results}

Expression and purification of a full-length recombinant SARS-CoV nsp12

The gene encoding the full-length nsp12 was amplified by RT-PCR using the total RNA from Vero cells infected with the SARS-CoV Urbani strain (Fig. 1A). We amplified the gene using a forward primer to generate an nsp12 gene that was in frame with the upstream ORF1a translation, resulting in an amino acid sequence that was the same at the N-terminus of SARS-CoV nsp12 (SADASTFLN). T7 RNA polymerase was previously shown to have an RNAlabeling activity in vitro on some RNAs through intra- or intermolecular priming [3]. Thus, to avoid potential contamination of T7 RNA polymerase, the amplified gene was expressed using the $E$. coli expression vector pTrcHisB, in which gene expression is controlled by a trc chimeric promoter. As shown in Fig. 1A, the recombinant nsp12 expressed from the resulting vector is expected to have extra amino acids at the $\mathrm{N}$-terminus, which are introduced during the cloning procedure. A (His) ${ }_{6}$-tag was also added 
A

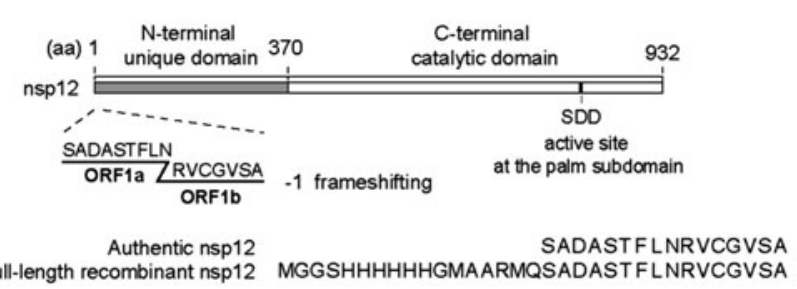

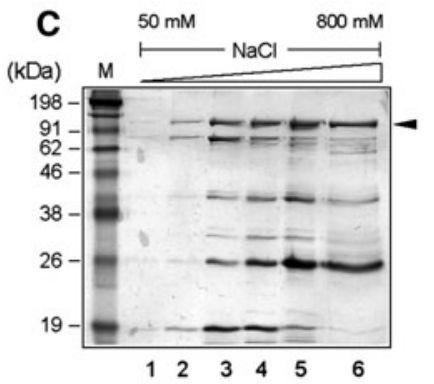

D
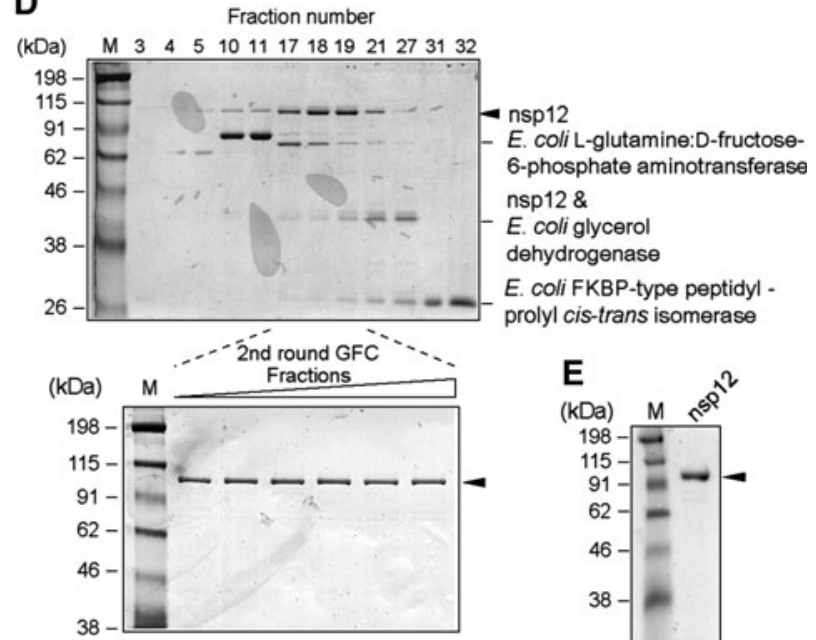

E

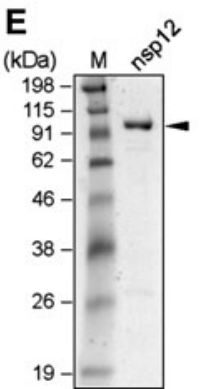

Fig. 1 Purification of full-length SARS-CoV nsp12 expressed in E. coli. (A) Schematic diagram of a full-length SARS-CoV nsp12. The unique domain, the catalytic domain, and the conserved RdRp active-site residues of the nsp12 are indicated. The deduced $\mathrm{N}$-terminal sequences of the indicated nsp12 protein are shown. (BD) The nsp12 protein was expressed in E. coli and purified by affinity chromatography using an Ni-NTA agarose column (B), anionexchange chromatography using a Q-Sepharose column $(\mathbf{C})$, and GFC using a Superdex 200 column (D). SARS-CoV nsp12 bound to an Ni-NTA agarose and a Q-Sepharose column were eluted by increasing concentrations of imidazole and $\mathrm{NaCl}$, respectively. Eluted proteins and fractions collected from the Superdex 200 column were separated by $10 \%$ SDS-PAGE and stained with Coomassie blue. Arrowheads indicate the position of SARS-CoV nsp12. In panel (D), proteins identified by MS/MS analysis are shown. (E) The final purified recombinant nsp12 $(2 \mu \mathrm{g})$ was resolved by SDS-PAGE and visualized by Coomassie blue staining

Nsp12 has primer- and $\mathrm{Mn}^{2+}$-dependent RdRp activity

Since divalent metal ions are essential cofactors for viral RdRps [13], we initially tested the RdRp activity of purified nsp12 in the presence of either $\mathrm{Mg}^{2+}$ or $\mathrm{Mn}^{2+}$. The RdRp assay was performed with a poly(A) RNA template in the absence and presence of the primer oligo(U) $)_{20}$. As shown in Fig. 2A, nsp12 exhibited primer-dependent RdRp activity that was strictly dependent on $\mathrm{Mn}^{2+}$ for RNA synthesis. $\mathrm{Mg}^{2+}$ could not replace $\mathrm{Mn}^{2+}(2 \mathrm{mM}$; Fig. 2B), even at concentrations up to $10 \mathrm{mM}$ (data not shown). Using the two viral-genome-derived RNA templates representing parts of the 3'-UTRs of the plus and minus strands of the viral genome, namely 3'-UTR339+16A and c5'-UTR119 (see the schematic diagrams of these RNA templates shown in Fig. 4), we consistently observed $\mathrm{Mn}^{2+}$-dependent RdRp activity (Fig. 2C). This $\mathrm{Mn}^{2+}$ dependent RdRp activity was also observed using a poly(C) template (Fig. 3B). The optimal $\mathrm{Mn}^{2+}$ concentration for nsp12 activity was $2 \mathrm{mM}$ (Fig. 2D). The temperature and $\mathrm{pH}$ for optimal SARS-CoV nsp12 activity were determined to be $32{ }^{\circ} \mathrm{C}$ and $\mathrm{pH} 7.5$, respectively (Fig. $2 \mathrm{E}$ and $\mathrm{F})$. Potassium $\left(\mathrm{K}^{+}\right)$ions exhibited an inhibitory effect on enzyme activity at concentrations higher than $50 \mathrm{mM}$ (Fig. 2G). 


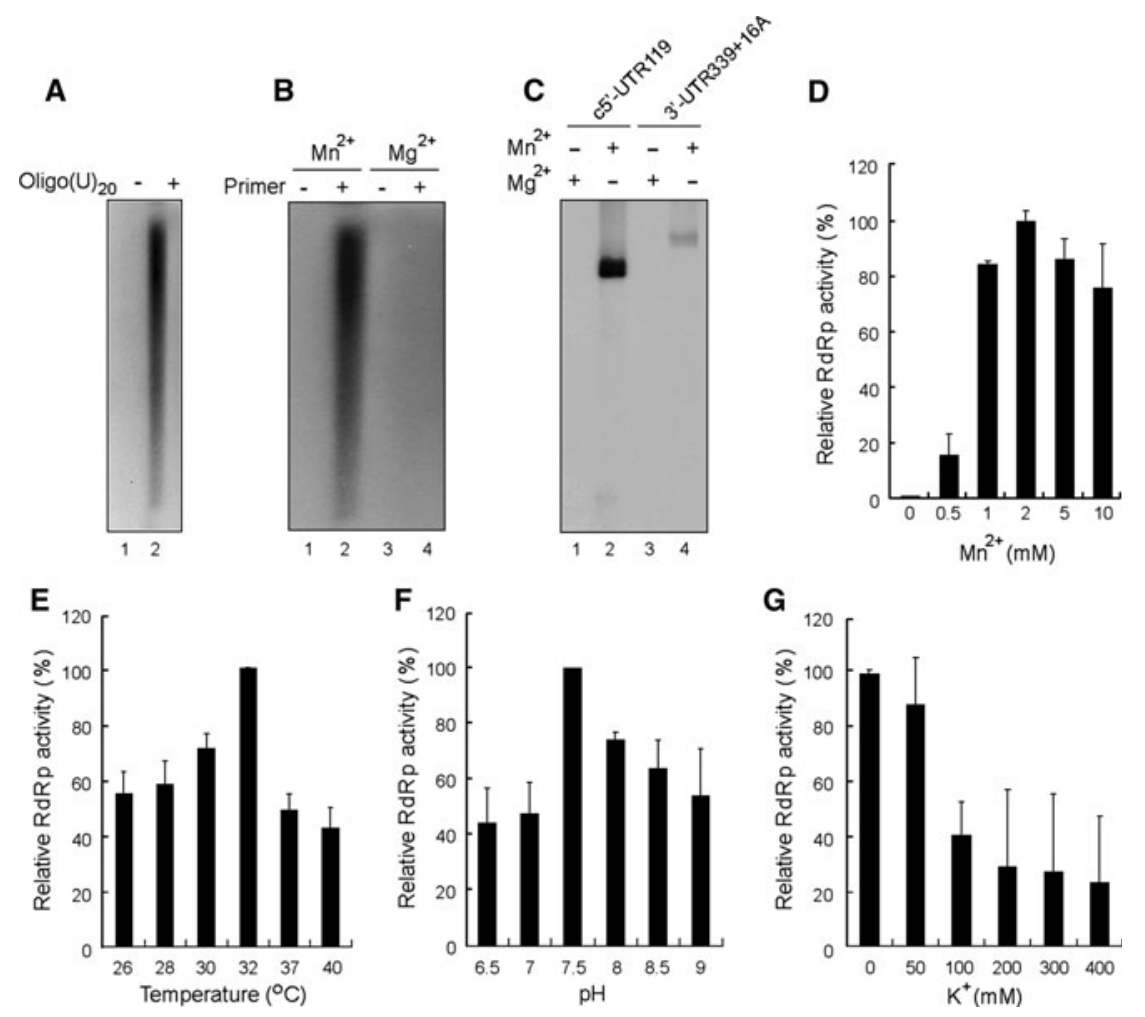

Fig. 2 Primer- and $\mathrm{Mn}^{2+}$-dependent RNA synthesis by nsp12. RdRp assays were performed with a homopolymeric poly(A) RNA template in the presence $(+)$ or absence $(-)$ of the 20-nt complementary ribonucleotide primer $\mathrm{U}_{20}(\mathbf{A}$ and $\mathbf{B}$, as indicated; $\mathbf{D}-\mathbf{G}$, in the presence of $U_{20}$ ) or with the viral-genome-derived RNA templates $(\mathbf{C}$, see schematic diagrams of the RNA templates in Fig. 4A and D). The reactions were carried out in the presence of $2 \mathrm{mM} \mathrm{Mn}^{2+}$ (A and
E-G), $2 \mathrm{mM} \mathrm{Mg}^{2+}$ or $\mathrm{Mn}^{2+}$ (B-C), or increasing concentrations of $\mathrm{Mn}^{2+}$ (D). Radioisotope-labeled RNA products were resolved on an $8 \mathrm{M}$ urea-5\% polyacrylamide gel before drying and being exposed to x-ray film for autoradiography. In (D-G), the relative RdRp activity of nsp12 is presented as the percentage of that obtained under each optimal condition
A

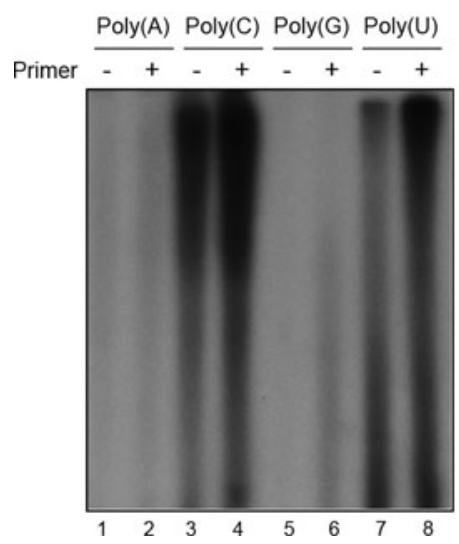

B

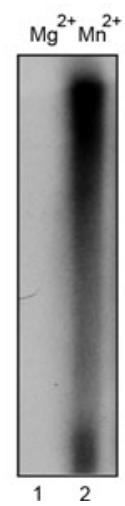

C

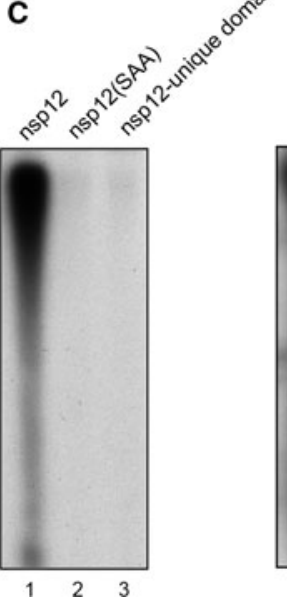

D

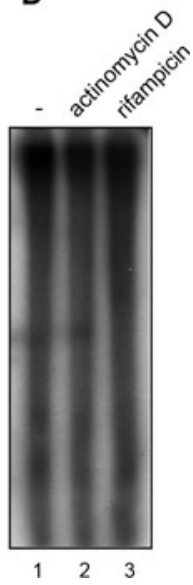

Fig. 3 Primer dependence of nsp12 in RNA synthesis initiation from homopolymeric RNA templates. (A) RdRp assays were performed using the indicated homopolymeric RNA templates in the absence $(-)$ or presence $(+)$ of 20-mer complementary ribonucleotide primer. $\mathrm{Mn}^{2+}(2 \mathrm{mM})$ was used as the metal cofactor. The amount of ${ }^{32} \mathrm{P}-\mathrm{GMP}$ incorporated in the reaction with the poly $(\mathrm{C}) /(\mathrm{rG})_{20}$ template was measured to be $\sim 5 \times 10^{4} \mathrm{cpm}$. (B) RdRp assays were performed with a poly $(\mathrm{C})$ template in the presence of $\mathrm{Mg}^{2+}$ or $\mathrm{Mn}^{2+}$.
(C) RdRp activities of wild-type nsp12, a mutant nsp12 carrying a SDD-to-SAA substitution [nsp12(SAA)], and the unique domain of nsp12 (nsp12-unique domain) were tested with a poly(C) template in the absence of the $(\mathrm{rG})_{20}$ primer. (D) RdRp assays with a poly $(\mathrm{C})$ template were carried out in the absence $(-)$ or presence of actinomycin D $(20 \mu \mathrm{g} / \mathrm{ml})$ or rifampicin $(20 \mu \mathrm{g} / \mathrm{ml})$. RNA products were analyzed as in Fig. 2 
RdRp activity of nsp12 on homopolymeric RNA templates

To investigate the template preference of the recombinant SARS-CoV nsp12, we performed RdRp assays with four different homopolymeric RNA templates in the absence or presence of 20-mer complementary ribonucleotide primers. Interestingly, we found that homopolymeric pyrimidine RNA templates $[\operatorname{poly}(\mathrm{C})$ and poly(U)] served as better templates than homopolymeric purine RNA templates [poly(A) and poly(G)] (Fig. 3A). The polymerase activity was highest with the poly $(\mathrm{C})$ template, and initiation of RNA synthesis occurred in the absence of a complementary $(\mathrm{rG})_{20}$ primer, although addition of the primer increased the levels of RNA products (compare lanes 3 and 4). A similar increase in the amount of labeled RNA product was observed using a poly(U) template with an (rA) 20 primer (lanes 7 and 8). In contrast, a low but detectable amount of RNA product was produced from the homopolymeric purine RNA templates only in the presence of complementary primers. Collectively, our results demonstrate that SARS-CoV nsp12 has both primer-dependent and primer-independent RNA synthesis activity, depending on the type of RNA template used.

The catalytic domain of SARS-CoV nsp12 is composed of finger, palm, and thumb subdomains, with a conserved SDD motif located in the active site of the palm subdomain (Fig. 1A) [39]. To prove that the RdRp activity of nsp12 is due to its own enzymatic activity, we mutated the $\mathrm{SDD}_{759-761}$ motif to SAA to inactivate the enzyme. As shown in Fig. 3C, while wild-type nsp12 had robust RdRp activity on a poly(C) template (lane 1), the SAA mutation completely abolished the polymerase activity (lane 2). SARS-CoV nsp12 has a unique domain encompassing the N-terminal 370 amino acids, which is not present in most other plus-strand RNA virus RdRps, and the functional importance of this domain has not been determined [29, 39]. We expressed and purified a recombinant form of the unique domain and determined whether it had RNA-labeling activity. Using the in vitro RdRp assay with a poly $(\mathrm{C})$ template, we found that the unique domain did not have RNA polymerase activity (Fig. 3C, lane 3 ). We also attempted to express the nsp12 catalytic domain (amino acids 371-932), but we could not get soluble recombinant proteins (data not shown). Lastly, the SARS-CoV RdRp activity was not significantly inhibited by either actinomycin $\mathrm{D}(20 \mu \mathrm{g} / \mathrm{ml})$ or rifampicin $(20 \mu \mathrm{g} / \mathrm{ml})$ (Fig. 3D), indicating that the RdRp activity of nsp12 was not attributable to contamination by bacterial DNA-dependent RNA polymerases.

Mapping of minimal cis-acting signals required for viral RNA synthesis initiation

It has been reported that the poly(A) tail is an important cis-acting element for coronavirus replication [30]. We sought to determine whether a poly(A) tail is required for in vitro minus-strand viral RNA synthesis. The 339-ntlong, full-length SARS-CoV 3'-UTR template lacking the poly(A) tail (Fig. 4A) and the 3'-UTR templates possessing different lengths of a poly(A) tail (13-32 polyadenylates) were prepared by in vitro transcription and used as templates for $\mathrm{RdRp}$ reactions. The recombinant nsp12 was able to copy the 3'-UTR template lacking the poly(A) tail in the absence of a primer (Fig. 4B, lane 1). Furthermore, the 3'-UTR RNA templates with 13-16 adenylates were used as efficiently as the 3'-UTR lacking the poly(A) tail (compare lane 1 with lanes 2 and 3). However, there was an incremental decrease in the amounts of RNA product when a poly(A) tail longer than $16 \mathrm{nt}$ was added to the template (lanes 4 and 5). Having found that the 3'-UTR upstream of the poly(A) tail is critical for viral minus-strand RNA synthesis, we mapped the minimal cis-acting RNA element within the 3'-UTR by RdRp assays with serial deletion derivatives of the 3'-UTR template lacking the poly(A) tail. As shown in Fig. 4C, nsp12 was able to synthesize templatesize labeled RNA products in a primer-independent manner, suggesting that a 36-nt sequence and/or a secondary structure in the 3'-UTR is a minimal RNA region recognized by nsp12 for de novo RNA synthesis initiation in vitro.

Like the 3'-UTR of the plus-strand viral genome, the 3'-end region of the minus-strand RNA template is also important for the initiation of viral RNA genome synthesis [21, 25]. To map the minimal region recognized by SARS-CoV RdRp, various RNA templates (Fig. 4D) representing the complementary sequences of the 5'-UTR of the SARS-CoV genome (c5'-UTR) were tested for their template activities in the in vitro $\mathrm{RdRp}$ assay. The $\mathrm{nsp} 12$ was able to initiate RNA synthesis with as little as $37 \mathrm{nt}$ of RNA from the 3' end of the c5'-UTR (Fig. 4E, lane 5). Thus, a 36-nt sequence from the 3'-UTR and a 37-nt sequence from the c5'-UTR, which were predicted to form stable stem-loop structures (Fig. 4A and D), seem to be the minimal cis-acting RNA elements required for nsp12 to initiate RNA synthesis. Together, the data suggested that nsp12 might be able to initiate RNA synthesis from either a double-stranded stem region (Fig. 4A) or a single-stranded region (Fig. 4D). Furthermore, these results clearly demonstrated that recombinant SARS-CoV nsp12 was able to initiate both minus- and plus-strand viral RNA synthesis in vitro without the aid of other viral and cellular proteins.

\section{Discussion}

In this study, we characterized the biochemical properties of a full-length SARS-CoV nsp12 that can initiate RNA synthesis de novo from the 3' ends of both the plus and 

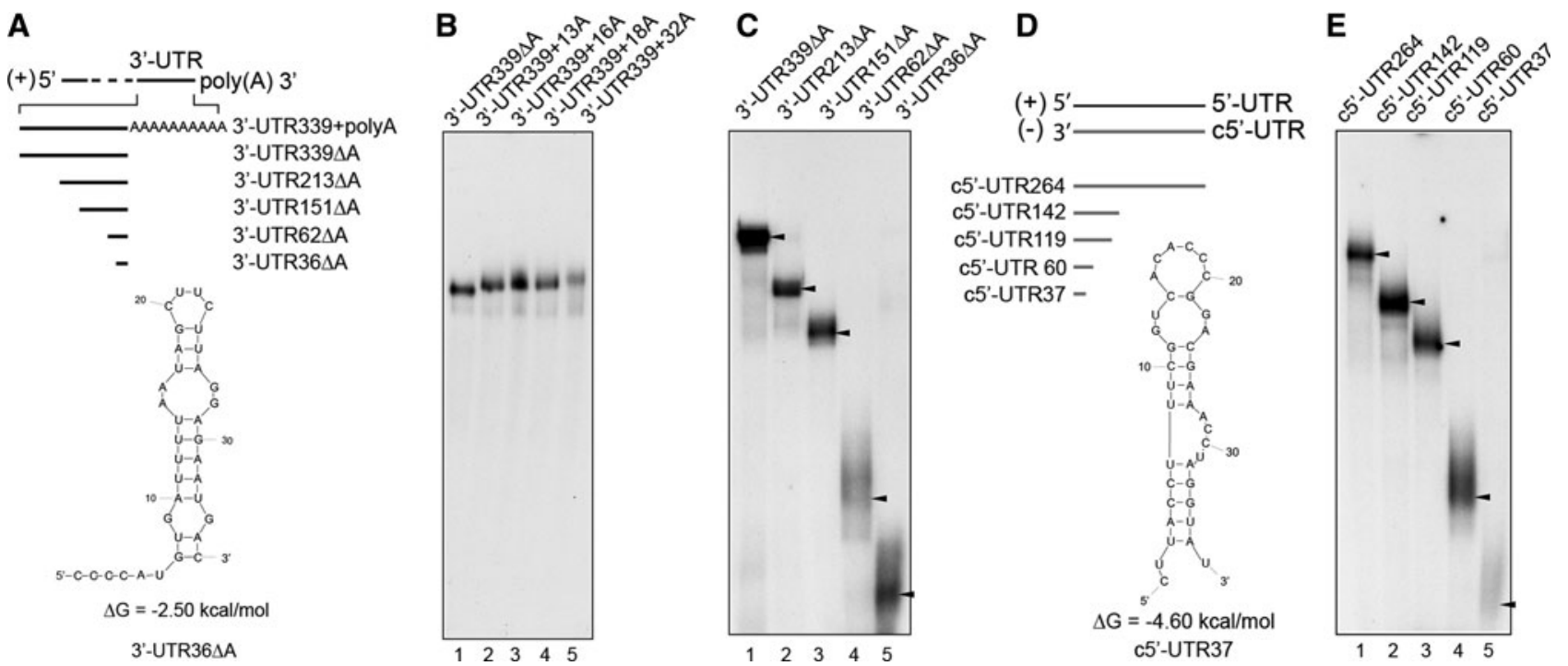

Fig. 4 Mapping of minimal RNA domains required for initiation of plus-strand and minus-strand viral RNA synthesis. (A) Schematic representation of 3'-UTR RNA templates without or with various lengths (13-32 nt) of polyadenylate and deletion derivatives of the 3'-UTR lacking a poly(A) tail (3'-UTR339 $\Delta$ A). The most stable secondary structure of 3'-UTR36 $\Delta$ A predicted by the Mfold program [40] is shown. (B) RdRp assays were performed with the indicated RNA templates. The numbers following the + sign of the series of 3'-UTR339 $\Delta$ A RNA templates indicate the length of the poly(A) tail. Radioisotope-labeled RNA products were resolved on a medium-size $8 \mathrm{M}$ urea- $8 \%$ acrylamide gel $(20 \mathrm{~cm} \times 20 \mathrm{~cm})$ and subjected to autoradiography. (C) Minimal cis-acting RNA elements required for minus-strand RNA synthesis were mapped by $\mathrm{RdRp}$

minus strands of the SARS-CoV genome. The nsp12 required a minimal region of less than $40 \mathrm{nt}$ at the termini of the viral genome and its complementary minus-strand RNA for initiation of RNA synthesis. Our data also demonstrated that the poly(A) tail at the 3' end of viral genome plays a regulatory role in initiation of RNA synthesis during minus-strand RNA synthesis.

Previously, SARS-CoV in vitro RdRp assay systems were established using a replication/transcription complex (RTC) [38] or using recombinant nsp12 [4, 34]. The RTC, which was isolated from double-membrane vesicles formed in SARS-CoV-infected cells, displayed an RdRp activity using endogenous RTC-associated viral RNA templates. However, it was not determined whether or not the SARS-CoV RTC could utilize exogenous RNA templates. Furthermore, the RdRp activity was dependent on an as-yet-unidentified host factor(s) present in the cytoplasmic soluble fraction. In contrast to the results observed with the RTC, a glutathione S-transferase (GST)-fused SARS-CoV RdRp showed primer-dependent RdRp activity on a poly(A) RNA template [4], which is consistent with our results. Another study also showed that the SARS-CoV nsp12 has primer-dependent RdRp activity on a synthetic RNA template consisting of a 20-nt duplex and a 5, assays using the indicated deletion derivatives of the 3'-UTR36 $\Delta$ A. The numbers following the letters " 3 '-UTR" indicate the corresponding size of each template. RNA products were analyzed as in (B). Template positions are indicated with arrowheads. (D) Deletion derivatives of c5'-UTR RNA templates are depicted on schematic diagrams. The most stable secondary structure of the c5'-UTR 38-nt RNA template predicted by the Mfold program is shown. The numbers following the letters "c5'-UTR" represent the corresponding size of each template. (E) Minimal cis-acting RNA elements required for plus-strand RNA synthesis were mapped by RdRp assays using the indicated RNA templates. Template positions are indicated by arrowheads

overhang of 10 uridylates, which could serve as a template during extension of the 20-nt primer [34]. However, none of the prior studies demonstrated bona fide RdRp activity of nsp12, i.e., RNA synthesis initiated de novo from exogenous RNA templates and from the 3' ends of viral genomic RNA and its complementary strand. We demonstrated for the first time that the recombinant nsp12 has RNA polymerase activity capable of initiating RNA synthesis de novo from the 3' ends of both plus and minus strands of the SARS-CoV genome. This nsp12 could also copy homopolymeric RNA templates in either a primerindependent manner with poly $(\mathrm{C})$ and poly $(\mathrm{U})$ templates or in a primer-dependent manner with poly(A) and poly $(\mathrm{G})$ templates. Our results also suggested that purine nucleotides are preferentially used by $\mathrm{nsp} 12$ for de novo initiation of RNA synthesis. In contrast, te Velthuis et al. [34] found that the use of $\operatorname{poly}(\mathrm{U})$ or poly $(\mathrm{C})$ template did not lead to significant incorporation of nucleotides in RdRp assays with nsp12 with a natural $\mathrm{N}$-terminus and a C-terminal hexahistidine tag. This dramatically different property of nsp12 might be in part attributed to the C-terminal hexahistidine tag.

In contrast to previous data obtained by using recombinant SARS-CoV RdRps [4, 34], our results also show 
that nsp12 strictly requires $\mathrm{Mn}^{2+}$ ions as a cofactor. Previously, the N-terminal GST-fused SARS-CoV nsp12 showed a primer-dependent RNA polymerase activity in the presence of $\mathrm{Mg}^{2+}$ using a poly(A)/oligoU $\mathrm{U}_{16}$ template [4]. However, the full-length nsp12 with a C-terminal hexahistidine tag displayed a primer-dependent RNA polymerase activity using a short primed poly(U) template in the presence of either $\mathrm{Mg}^{2+}$ or $\mathrm{Mn}^{2+}$ [33]. The authors demonstrated that $\mathrm{Mn}^{2+}$ promoted misincorporation of ribonucleotides. The SARS-CoV RTC complex isolated from virus-infected cells displayed RdRp activities in vitro only in the presence of $\mathrm{Mg}^{2+}$ ions [14]. The reason for the requirement of different metal ions by the RTC and recombinant nsp12 proteins is not clear. It is possible that the hexahistidine tag or fused proteins at the $\mathrm{N}$ - or C-terminus of nsp12 might affect the enzymatic properties of the recombinant nsp12 proteins. Nevertheless, it is worth noting that SARS-CoV nsp8, a second non-canonical RdRp, also requires $\mathrm{Mn}^{2+}$ as a cofactor [10]. Since nsp8 interacts directly with nsp12, as demonstrated by GST pull-down experiments [10], these two proteins may form a replicase complex showing $\mathrm{Mn}^{2+}$ ion dependence for RNA synthesis. It has been argued that $\mathrm{Mn}^{2+}$ is unlikely to act as a cofactor of cellular and viral enzymes because $\mathrm{Mn}^{2+}$ is known to be present at less than $1 \%$ of the abundance of $\mathrm{Mg}^{2+}$ [24]. However, various enzymes, including viral RdRps, have shown a strict $\mathrm{Mn}^{2+}$-dependence [24]. $\mathrm{Mn}^{2+}$ dependent RdRps might overcome the limited availability of $\mathrm{Mn}^{2+}$ by their high affinity for $\mathrm{Mn}^{2+}$. It is equally plausible to speculate that SARS-CoV nsp12, like other viral RNA polymerases [9, 24, 33], becomes more errorprone with $\mathrm{Mn}^{2+}$. The dependence on $\mathrm{Mn}^{2+}$ ions for $\mathrm{RdRp}$ activity was also observed for other viral RdRps, including Japanese encephalitis virus NS5 RdRp [13], equine arteritis virus nsp9 RdRp [2], RdRps of dengue virus and West Nile virus [27], and hepatitis C virus NS5B RdRp [5, 17, 24]. Recent studies showed that HCV NS5B displays different modes of RNA synthesis initiation depending on the types of catalytic metal ions, $\mathrm{Mg}^{2+}$ or $\mathrm{Mn}^{2+} ; \mathrm{Mn}^{2+}$ appears to stimulate de novo RNA synthesis on certain RNA templates [24].

Stable RNA secondary structures located at the 3' termini of single-stranded RNA viruses play an important role in the initiation of genomic replication $[8,16,20,22]$. In addition, the poly(A) tail has been proposed as a potential cis-acting element for genome replication and transcription in several viruses [15, 31, 32, 36]. However, it still remains to be elucidated whether an increase of viral genome abundance after the addition of a poly(A) tail to the 3 '-end of viral genomes or defective interfering RNAs was due to the direct effect on viral RNA synthesis rather than to the enhancement of viral gene expression or RNA genome stabilization. Our results demonstrated that the poly(A) tail of the SARS-CoV genome is dispensable for the initiation of minus-strand viral RNA synthesis. Although the SARS$\mathrm{CoV}$ nsp12 required a primer to direct RNA synthesis using a homopolymeric poly(A) template, the tailless 3'-UTR terminal 36-nt served as a template for the de novo initiation of RNA synthesis. Thus, the 3'-end RNA structure and/or sequence upstream of the poly(A) tail appears to be the major cis-acting element required for initiation of minus-strand RNA synthesis and possibly for discrimination between cellular mRNAs and the viral genomic and subgenomic RNAs. Our data also showed that minus-strand RNA synthesis is inhibited by a poly(A) tail longer than 16 nt, suggesting a regulatory role of the poly(A) in RNA synthesis. Thus, it will be of interest to test whether SARS$\mathrm{CoV}$ genomic and subgenomic RNAs carrying poly(A) tail of different lengths can be differentially utilized by the viral RNA replicase and translational machinery.

Even though the terminal 36-nt of the 3'-UTR allowed nsp12 to carry out de novo initiation of RNA synthesis in vitro, it remains to be determined whether other viral or cellular proteins are required in vivo for the SARS-CoV replicase to recognize specific promoter sequences on the SARS-CoV 3'-UTR. For instance, as proposed recently by Zust et al. [41] for mouse hepatitis virus (MHV) minusstrand RNA synthesis, nsp8 primase of SARS-CoV might bind to a 3'-UTR stem-loop structure and initiate minusstrand RNA synthesis by production of a tract of oligo(U) using the 3'-end poly(A) tail of the viral genome as a template. The authors proposed that the synthesis of a short primer representing the 5' end of minus-strand RNA leads to separation of the 3' end of the genome from pseudoknot loop 1, allowing the formation of a completely folded pseudoknot. That structure, together with nsp8 and other viral nonstructural proteins, then recruits nsp12 to resume elongation of minus-strand RNA synthesis by primer-extension. This notion is in part supported by the fact that the SARS-CoV nsp8 displays an RdRp activity synthesizing a short oligonucleotide [10]. Thus, nsp8 of SARS-CoV may be required to form a pre-initiation RNA replicase complex through interaction with nsp12 [10]. Direct binding of an nsp8/nsp12 complex or sequential binding of nsp8 and nsp12 to the 3'-UTR may cooperatively carry out minus-strand RNA synthesis. It would be of interest to investigate whether a short primer generated by the primase activity of nsp8 can be in turn extended by the primer-dependent RNA polymerase activity of nsp12. Furthermore, it should be also noted that coronaviruses, including MHV, bovine coronavirus, and SARS-CoV, have two structurally conserved RNA structures, a bulged stemloop and an adjacent pseudoknot, upstream of the 3'-UTR [7]. Genetic studies using a full-length MHV-A59 cDNA cloned into vaccinia virus showed a genetic interaction of the loop 1 of the pseudoknot with the nsp8 and nsp4, which 
are the components of the RNA replicase [41]. Since the SARS-CoV 3'-UTR was able to functionally replace its counterpart in the MHV genome, the pseudoknot region might be a critical cis-acting element for viral genome replication in vivo. Further analysis of the roles of the 3'UTR and viral or cellular proteins interacting with this cisacting RNA in RNA synthesis initiation is warranted using the in vitro RdRp assay system we have established in the present study.

In summary, we have characterized the biochemical properties of a functionally active, full-length SARS-CoV nsp12. The in vitro RdRp assay system will be useful for understanding the mechanism of SARS-CoV replication, including how subgenomic viral RNAs are synthesized and what viral and/or host factors are involved in RNA synthesis as cofactors. In addition, the recombinant nsp12 capable of copying the SARS-CoV genome-derived RNA templates will provide a valuable tool for discovery of antiviral drugs against SARS-CoV.

Acknowledgments This work was supported by the Seoul R\&BD Program (Grant 10580), Technology Innovation Program (MKE 10035159) funded by the Ministry of Knowledge Economy (MKE, Korea), and in part by grants from the National Research Foundation of Korea (NRF-2011-0001236, 2011-0027650, and 2010-0029116).

\section{References}

1. Ahn DG, Lee W, Choi JK, Kim SJ, Plant EP, Almazan F, Taylor DR, Enjuanes L, Oh JW (2011) Interference of ribosomal frameshifting by antisense peptide nucleic acids suppresses SARS coronavirus replication. Antiviral Res 91:1-10

2. Beerens N, Selisko B, Ricagno S, Imbert I, van der Zanden L, Snijder EJ, Canard B (2007) De novo initiation of RNA synthesis by the arterivirus RNA-dependent RNA polymerase. J Virol 81:8384-8395

3. Cazenave C, Uhlenbeck OC (1994) RNA template-directed RNA synthesis by T7 RNA polymerase. Proc Natl Acad Sci USA 91:6972-6976

4. Cheng A, Zhang W, Xie Y, Jiang W, Arnold E, Sarafianos SG, Ding J (2005) Expression, purification, and characterization of SARS coronavirus RNA polymerase. Virology 335:165-176

5. Clemente-Casares P, Lopez-Jimenez AJ, Bellon-Echeverria I, Encinar JA, Martinez-Alfaro E, Perez-Flores R, Mas A (2011) De novo polymerase activity and oligomerization of hepatitis $C$ virus RNA-dependent RNA-polymerases from genotypes 1 to 5. PLoS One 6:e18515

6. De Rijk P, Wuyts J, De Wachter R (2003) RnaViz 2: an improved representation of RNA secondary structure. Bioinformatics 19:299-300

7. Goebel SJ, Taylor J, Masters PS (2004) The 3' cis-acting genomic replication element of the severe acute respiratory syndrome coronavirus can function in the murine coronavirus genome. J Virol 78:7846-7851

8. Hong Z, Cameron CE, Walker MP, Castro C, Yao N, Lau JY, Zhong W (2001) A novel mechanism to ensure terminal initiation by hepatitis C virus NS5B polymerase. Virology 285:6-11

9. Huang Y, Beaudry A, McSwiggen J, Sousa R (1997) Determinants of ribose specificity in RNA polymerization: effects of
$\mathrm{Mn}^{2+}$ and deoxynucleoside monophosphate incorporation into transcripts. Biochemistry 36:13718-13728

10. Imbert I, Guillemot JC, Bourhis JM, Bussetta C, Coutard B, Egloff MP, Ferron F, Gorbalenya AE, Canard B (2006) A second, non-canonical RNA-dependent RNA polymerase in SARS coronavirus. EMBO J 25:4933-4942

11. Jacobson AB, Zuker M (1993) Structural analysis by energy dot plot of a large mRNA. J Mol Biol 233:261-269

12. Kao CC, Singh P, Ecker DJ (2001) De novo initiation of viral RNA-dependent RNA synthesis. Virology 287:251-260

13. Kim YG, Yoo JS, Kim JH, Kim CM, Oh JW (2007) Biochemical characterization of a recombinant Japanese encephalitis virus RNA-dependent RNA polymerase. BMC Mol Biol 8:59

14. Knoops K, Kikkert M, Worm SH, Zevenhoven-Dobbe JC, van der Meer Y, Koster AJ, Mommaas AM, Snijder EJ (2008) SARScoronavirus replication is supported by a reticulovesicular network of modified endoplasmic reticulum. PLoS Biol 6:e226

15. Kusov YY, Gosert R, Gauss-Muller V (2005) Replication and in vivo repair of the hepatitis A virus genome lacking the poly(A) tail. J Gen Virol 86:1363-1368

16. Larsen GR, Semler BL, Wimmer E (1981) Stable hairpin structure within the 5'-terminal 85 nucleotides of poliovirus RNA. J Virol 37:328-335

17. Luo G, Hamatake RK, Mathis DM, Racela J, Rigat KL, Lemm J, Colonno RJ (2000) De novo initiation of RNA synthesis by the RNA-dependent RNA polymerase (NS5B) of hepatitis C virus. J Virol 74:851-863

18. Marra MA, Jones SJ, Astell CR, Holt RA, Brooks-Wilson A, Butterfield YS, Khattra J, Asano JK, Barber SA, Chan SY, Cloutier A, Coughlin SM, Freeman D, Girn N, Griffith OL, Leach SR, Mayo M, McDonald H, Montgomery SB, Pandoh PK, Petrescu AS, Robertson AG, Schein JE, Siddiqui A, Smailus DE, Stott JM, Yang GS, Plummer F, Andonov A, Artsob H, Bastien N, Bernard K, Booth TF, Bowness D, Czub M, Drebot M, Fernando L, Flick R, Garbutt M, Gray M, Grolla A, Jones S, Feldmann H, Meyers A, Kabani A, Li Y, Normand S, Stroher U, Tipples GA, Tyler S, Vogrig R, Ward D, Watson B, Brunham RC, Krajden M, Petric M, Skowronski DM, Upton C, Roper RL (2003) The Genome sequence of the SARSassociated coronavirus. Science 300:1399-1404

19. Masters PS (2006) The molecular biology of coronaviruses. Adv Virus Res 66:193-292

20. Nagy PD, Simon AE (1998) In vitro characterization of late steps of RNA recombination in turnip crinkle virus. I. Role of motif1hairpin structure. Virology 249:379-392

21. Oh JW, Ito T, Lai MM (1999) A recombinant hepatitis C virus RNA-dependent RNA polymerase capable of copying the fulllength viral RNA. J Virol 73:7694-7702

22. Paul AV, Rieder E, Kim DW, van Boom JH, Wimmer E (2000) Identification of an RNA hairpin in poliovirus RNA that serves as the primary template in the in vitro uridylylation of VPg. J Virol 74:10359-10370

23. Plant EP, Rakauskaite R, Taylor DR, Dinman JD (2010) Achieving a golden mean: mechanisms by which coronaviruses ensure synthesis of the correct stoichiometric ratios of viral proteins. J Virol 84:4330-4340

24. Ranjith-Kumar CT, Kim YC, Gutshall L, Silverman C, Khandekar S, Sarisky RT, Kao CC (2002) Mechanism of de novo initiation by the hepatitis $\mathrm{C}$ virus RNA-dependent RNA polymerase: role of divalent metals. J Virol 76:12513-12525

25. Reigadas S, Ventura M, Sarih-Cottin L, Castroviejo M, Litvak S, Astier-Gin T (2001) HCV RNA-dependent RNA polymerase replicates in vitro the 3' terminal region of the minus-strand viral RNA more efficiently than the 3' terminal region of the plus RNA. Eur J Biochem 268:5857-5867

26. Sawicki SG, Sawicki DL, Siddell SG (2007) A contemporary view of coronavirus transcription. J Virol 81:20-29 
27. Selisko B, Dutartre H, Guillemot JC, Debarnot C, Benarroch D, Khromykh A, Despres P, Egloff MP, Canard B (2006) Comparative mechanistic studies of de novo RNA synthesis by flavivirus RNA-dependent RNA polymerases. Virology 351:145-158

28. Shevchenko A, Wilm M, Vorm O, Mann M (1996) Mass spectrometric sequencing of proteins silver-stained polyacrylamide gels. Anal Chem 68:850-858

29. Snijder EJ, Bredenbeek PJ, Dobbe JC, Thiel V, Ziebuhr J, Poon LL, Guan Y, Rozanov M, Spaan WJ, Gorbalenya AE (2003) Unique and conserved features of genome and proteome of SARS-coronavirus, an early split-off from the coronavirus group 2 lineage. J Mol Biol 331:991-1004

30. Spagnolo JF, Hogue BG (2000) Host protein interactions with the 3 ' end of bovine coronavirus RNA and the requirement of the poly(A) tail for coronavirus defective genome replication. J Virol 74:5053-5065

31. Spagnolo JF, Hogue BG (2001) Requirement of the poly(A) tail in coronavirus genome replication. Adv Exp Med Biol 494:467-474

32. Svitkin YV, Costa-Mattioli M, Herdy B, Perreault S, Sonenberg $N$ (2007) Stimulation of picornavirus replication by the poly(A) tail in a cell-free extract is largely independent of the poly(A) binding protein (PABP). RNA 13:2330-2340

33. te Velthuis AJ, Arnold JJ, Cameron CE, van den Worm SH, Snijder EJ (2010) The RNA polymerase activity of SARS-coronavirus nsp12 is primer dependent. Nucleic Acids Res 38:203-214

34. te Velthuis AJ, Arnold JJ, Cameron CE, van den Worm SH, Snijder EJ (2010) The RNA polymerase activity of SARS-coronavirus nsp12 is primer dependent. Nucleic Acids Res 38:203-214
35. Thiel V, Ivanov KA, Putics A, Hertzig T, Schelle B, Bayer S, Weissbrich B, Snijder EJ, Rabenau H, Doerr HW, Gorbalenya AE, Ziebuhr J (2003) Mechanisms and enzymes involved in SARS coronavirus genome expression. J Gen Virol 84:23052315

36. Tsai CH, Cheng CP, Peng CW, Lin BY, Lin NS, Hsu YH (1999) Sufficient length of a poly(A) tail for the formation of a potential pseudoknot is required for efficient replication of bamboo mosaic potexvirus RNA. J Virol 73:2703-2709

37. van Dijk AA, Makeyev EV, Bamford DH (2004) Initiation of viral RNA-dependent RNA polymerization. J Gen Virol 85: 1077-1093

38. van Hemert MJ, van den Worm SH, Knoops K, Mommaas AM, Gorbalenya AE, Snijder EJ (2008) SARS-coronavirus replication/ transcription complexes are membrane-protected and need a host factor for activity in vitro. PLoS Pathog 4:e1000054

39. Xu X, Liu Y, Weiss S, Arnold E, Sarafianos SG, Ding J (2003) Molecular model of SARS coronavirus polymerase: implications for biochemical functions and drug design. Nucleic Acids Res 31:7117-7130

40. Zuker M (2003) Mfold web server for nucleic acid folding and hybridization prediction. Nucleic Acids Res 31:3406-3415

41. Zust R, Miller TB, Goebel SJ, Thiel V, Masters PS (2008) Genetic interactions between an essential 3' cis-acting RNA pseudoknot, replicase gene products, and the extreme 3' end of the mouse coronavirus genome. J Virol 82:1214-1228 